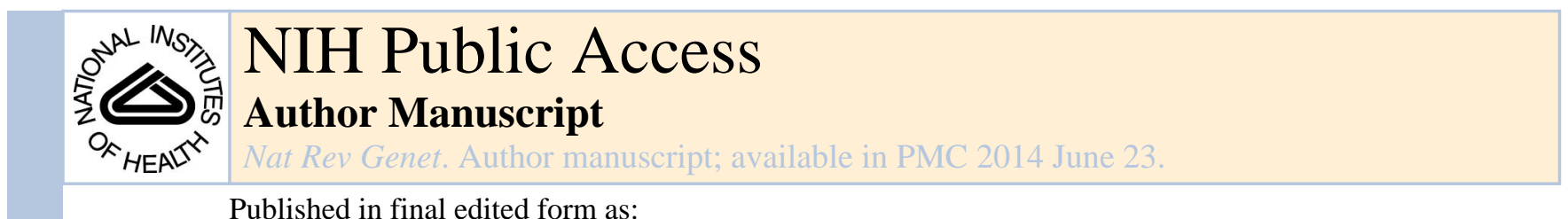

Published in final edited form as:

Nat Rev Genet. 2013 March ; 14(3): 228-235. doi:10.1038/nrg3435.

\title{
Transgenerational epigenetic inheritance: how important is it?
}

\author{
Ueli Grossniklaus, \\ Institute of Plant Biology and Zürich-Basel Plant Science Center, University of Zürich, \\ Zollikerstrasse 107, CH-8008 Zürich, Switzerland. grossnik@botinst.uzh.ch \\ William G. Kelly, \\ Biology Department, Emory University, 1510 Clifton Road NE, Atlanta, Georgia 30322, USA. \\ bkelly@emory.edu
}

Anne C. Ferguson-Smith, Department of Physiology, Development and Neuroscience and the Centre for Trophoblast Research, University of Cambridge, Downing Street, Cambridge CB2 3EG, UK. afsmith@mole.bio.cam.ac.uk

Marcus Pembrey, and Clinical and Molecular Genetics Unit, Institute of Child Health, University College London, London WC1N 1EH, UK; Avon Longitudinal Study of Parents and Children, School of Social and Community Medicine, Oakfield House, Oakfield Grove, University of Bristol, Bristol BS8 2BN, UK. m.pembrey@bristol.ac.uk

\section{() 2013 Macmillan Publishers Limited. All rights reserved}

\section{The contributors}

Ueli Grossniklaus is Professor of Plant Developmental Genetics at the Institute of Plant Biology of the University of Zürich, Switzerland. From 2006 to 2011, he was President of the Zürich-Basel Plant Science Center and is currently Co-leader of the University Research Priority Program 'Evolution in Action'. His research focuses on the genetic and molecular basis of plant reproduction. He made seminal discoveries on the mechanisms that epigenetically control seed development both through maternally deposited factors and by genomic imprinting, a paradigm for epigenetic regulation. Over the past years, ecological and evolutionary aspects have been included in his research with the goal of gaining a better understanding of the role of epigenetics in adaptation. William G. Kelly is the Director of the Genetics and Molecular Biology Program and an associate professor in the Biology Department at Emory University, Atlanta, Georgia, USA. His research has focused on epigenetic regulation of germ cell specification, maintenance and differentiation, and epigenetic memory and reprogramming using Caenorhabditis elegans as a model system. His research has been supported by the US National Institutes of General Medical Sciences in the National Institutes of Health.

Anne C. Ferguson-Smith is Professor of Developmental Genetics in the Department of Physiology, Development and Neuroscience at the University of Cambridge, UK. For the past two decades, her team has studied genomic imprinting in development and disease and the epigenetic control of genome function in a wider context. Her current research focuses on three themes: stem cells and the epigenetic programme, functional epigenomics, and development environment and disease.

Marcus Pembrey is Emeritus Professor of Paediatric Genetics at the Institute of Child Health, University College London, UK, and a visiting professor of Paediatric Genetics at the University of Bristol, UK. Marcus was previously an honorary consultant clinical geneticist at Great Ormond Street Hospital for Children, London, UK. In 1988, he helped Jean Golding to establish the Avon Longitudinal Study of Parents and Children (ALSPAC) in Bristol, UK, and served as its Director of Genetics until 2005. Interested in unusual inheritance patterns, he studied fragile X and Angelman syndrome during 1980-1990 and then moved onto study epigenetics more generally. His current research focus is on environmental epigenomics and human transgenerational responses. Susan Lindquist has shown that the forces governing protein folding can have a profound and unexpected impact on evolution and human disease. She discovered the disaggregating abilities of heat-shock proteins, identified prions as conduits of protein-based inheritance and pioneered the use of yeast as a model system to study complex diseases and to develop novel therapeutic strategies. She found that protein-folding processes buffer and release the effects of accumulated genetic variation, providing the first plausible explanation for rapid bursts of evolution and established that the heat-shock response has a key role in the evolution of fungal drug resistance and tumour progression.

Competing interests statement

The authors declare no competing financial interests. 


\section{Susan Lindquist \\ Howard Hughes Medical Institute, Whitehead Institute for Biomedical Research, Massachusetts Institute of Technology, Nine Cambridge Center, Cambridge, Massachusetts 02142, USA. lindquist_admin@wi.mit.edu}

\section{Abstract}

Much attention has been given to the idea of transgenerational epigenetic inheritance, but fundamental questions remain regarding how much takes place and the impact that this might have on organisms. We asked five leading researchers in this area - working on a range of model organisms and in human disease - for their views on these topics. Their responses highlight the mixture of excitement and caution that surrounds transgenerational epigenetic inheritance and the wide gulf between species in terms of our knowledge of the mechanisms that may be involved.

Q How much transgenerational epigenetic inheritance takes place?

Ueli Grossniklaus. To give a short answer first: I do not think we know how widespread transgenerational epigenetic inheritance is in any organism. Given this uncertainty, it is surprising how much attention this question has attracted in recent years, both in the popular press and in the scientific literature (for example, see REFS 1-3). In principle, many inherited traits could have an epigenetic basis, as one cannot easily distinguish whether a phenotype is due to genetic or epigenetic variation without a detailed molecular analysis. It is also possible that epigenetically controlled traits are rare. But given the rapid advance of epigenomics, I am sure that we will get a much better understanding of the contribution of genetic versus epigenetic variation to phenotypes in the near future.

To provide a more profound answer, however, I will have to define what I understand by 'transgenerational' and 'epigenetic', given that the use of both terms varies widely. A popular definition of epigenetics states that it concerns the study of mitotically and/or meiotically heritable changes in gene expression that occur without a change in DNA sequence ${ }^{4}$. Under this definition, epigenetic regulation has a role at two levels. First, it is involved in development, leading to the specification of cells and assuring the faithful inheritance of their differentiated state over mitotic cell divisions. Second, epigenetic states can be inherited meiotically, from one generation to the next, and this is the focus here.

While some biologists consider all effects that concern both parents and offspring to be transgenerational, I would like to distinguish transgenerational effects from parental and depending on the system - grandparental effects. In addition to contributing their DNA, parents can influence their offspring in many ways: for example, by contributing bioactive molecules in the egg and sperm cytoplasm, by providing nutrients and hormonal information during embryogenesis and by provisioning and taking care of offspring after birth.

"when considering environmentally induced effects ... an epigenetic basis can be inferred only if they last over multiple generations"

Many of these parental (and sometimes grandparental) effects will not have an epigenetic basis. This is particularly important when considering environmentally induced effects, for 
which an epigenetic basis can be inferred only if they last over multiple generations. In a pregnant mammal, for instance, not only are the mother and fetus exposed to the same environmental influences but so are the fetus's primordial germ cells, which will eventually produce the grandchildren. For instance, expression of the methylation-sensitive, metastable agouti viable yellow $\left(A^{v y}\right)$ allele, which determines mouse coat colour and shows meiotic epigenetic inheritance ${ }^{5}$, is modulated by the animal's diet ${ }^{6}$. The specific dietary conditions in which a pregnant female is raised can change the inheritance pattern over two generations, but this change gets lost in the third generation ${ }^{7}$. Thus, while this specific diet leads to parental and grandparental effects, the induced epigenetic changes are not transgenerationally inherited.

Often ignored in studies of transgenerational inheritance, parental effects can also occur in plants. The condition of the mother plant has a strong effect on the seeds it produces with respect to, for instance, nutrient provisioning. At the time of dispersal, the offspring itself is still surrounded by the maternal tissues of the seed coat, and these tissues play a crucial part in the hormonal regulation of seed germination. Thus, in plants too, parental effects can have a strong effect on the next generation ${ }^{8}$ and may confound studies of epigenetic inheritance ${ }^{9}$.

In many experimental systems, there is clear evidence that silenced transgenes are stably inherited over several generations. This is different for natural epigenetic variants that cause a phenotype (that is, epialleles). In mammals, only very few - sometimes controversial cases of meitotically inherited epialleles have been reported, mostly with a variable degree of inheritance ${ }^{3}$. By contrast, there are several well-studied examples of stably inherited epialleles in plants ${ }^{10}$, of which I will mention just two. The first example was reported in toadflax (Linaria vulgaris), in which a variant with radially symmetric flowers, rather than the typical bilaterally symmetric ones, was already described by Lineus nearly 200 years ago. This phenotype is not caused by a mutation but by DNA methylation of the CYCLOIDEA gene, which controls the formation of dorsal petals ${ }^{11}$. Another example of a natural epiallele causes the Colourless non-ripening phenotype in tomatoes (Solanum lycopersicum $)^{12}$. In both cases, no differences in DNA sequence could be detected at the affected loci, strongly indicating that the mutant phenotypes had an epigenetic nature.

William G. Kelly. Research in Caenorhabditis elegans has illustrated numerous instances of transgenerational epigenetic inheritance. Examples of epigenomic patterns that are stably inherited include those that have been observed in other species (for example, heterochromatin and centromeric histone patterns) but also epigenomic patterns that are established by gene activity.

The heritable silencing of repetitive DNA elements - the heterochromatic compartment in many genomes - is clearly transgenerational and involves epigenetic mechanisms. Recent work from multiple laboratories has shown that the highly conserved PIWI-interacting RNA (piRNA) system (which involves a specific class of small, non-coding RNAs) acts in the $C$. elegans germ line as a sort of adaptive genome immunity system, in which an enormous diversity of RNA sequences can potentially be recognized and targeted for assault (for example, see REFS 13,14). The initiating response is guided by piRNAs, from which a secondary system refines and amplifies the response, narrowing the target specificity and 
increasing the efficiency. Importantly, almost any sequence can be identified — not just repetitive elements - and thus endogenous loci can also be targeted by this mechanism in an autoimmune response. Targeting of 'self' sequences appears to be prevented both by selection against selftargeting piRNAs and by a counteracting process using RNAs that are generated through normal germ cell transcripts (that is, self RNAs). Successful and heritable repression is achieved though the assembly of repressive chromatin, which can be stable for many generations. None of this multi-generational repression involves a genetic change in the targeted sequences.

These observations represent the repressive side of transgenerational epigenetic inheritance: there is also the opportunity for heritable activation of genes. Transcription in the germ line has the capability not only to generate self RNAs to prevent piRNA targeting but also to alter chromatin by mobilizing nucleosomes and producing modified chromatin in its wake. C. elegans embryonic chromatin carries an epigenetic memory of transcription that last occurred in the parental germ cells ${ }^{15,16}$. This memory includes covalent modifications (histone H3 methylation isoforms) that are associated with 'open' chromatin and added to genes during transcription in the parent. The methylation patterns are subsequently maintained in the embryo by methyltransferases that can operate independently of transcription. The presence of these particular marks can be antagonistic to other repressive histone $\mathrm{H} 3$ methylation marks. Thus, transcription-coupled establishment and maintenance of histone marks in germ cell chromatin, perhaps coupled with the insertion of histone variants, can prevent intrusion of repressive chromatin assembly into genes, and this appears to be required to promote the proper activation of these genes in the next generation ${ }^{17}$.

Indeed, the clearest example of transgenerational epigenetic inheritance in many organisms, centromeric chromatin, also appears to be guided by transcription in C. elegans, albeit negatively. The heritably stable positions of centromeres are guided by incorporation of the centromere-specific histone H3 variant CENPA (also known as CenH3). CENPA incorporation, which produces the $C$. elegans holocentromere, occurs where transcription activity is absent in parental germ cells; that is, transcription prevents stable CENPA placement in parental germ cell chromatin, and this heritably templates stable centromeric chromatin assembly in the offspring ${ }^{18}$.

Therefore, an answer to 'how much transgenerational inheritance takes place' in C. elegans may be that this seems to be a guiding mechanism for centromere designation and much of the germline transcriptome in this organism. It is worth noting that most of the factors that guide these epigenetic processes in C. elegans have orthologues in most eukaryotes, so it is certainly plausible that these routes to epigenetic inheritance exist in many organisms.

Anne C. Ferguson-Smith. The impact of the environment has been observed to extend over multiple generations in both human populations and animal models ${ }^{19-23}$, suggesting transgenerational epigenetic effects. However, a defined mechanism for such inheritance is missing.

It is clear that epigenetics can confer stable, heritable, functional genomic configurations within somatic lineages ${ }^{24,25}$. However, one of the most important properties of this 
epigenetic memory is its dynamic nature and particularly its ability to be erased ${ }^{26}$. This erasure happens both in the germ line and early in pre-implantation mammalian development and is followed by the establishment of new functional chromatin configurations. If true epigenetic inheritance is to occur, then under particular circumstances some epigenetic marks would be expected to be completely or partially resistant to both of these reprogramming events ${ }^{27}$.

At unique sequences, strong evidence that DNA methylation is a transgenerationally transmitted heritable epigenetic mark remains elusive. However, it may be no coincidence that many cases of transgenerational epigenetic inheritance involve repeat sequences ${ }^{3}$. Epigenetic silencing mechanisms are likely to have evolved, at least in part, to repress repetitive elements that have the potential to activate and destabilize the genome and/or to change the expression of adjacent genes ${ }^{28}$. Hence, these elements are a target for the epigenetic machinery in somatic tissues and especially in the germ line. In particular, DNA methylation of repetitive retrotransposons has provided one of the best models of transgenerational epigenetic inheritance. For example, the $A^{v y}$ allele in mice is an insertion of a long terminal repeat (LTR) retrotransposon called an intracisternal A-type particle (IAP) upstream of the agouti coat colour gene. Trangenerational inheritance of coat colour occurs, which depends on the extent of methylation of this element ${ }^{5}$. In addition, methylation of $A^{v y}$ in offspring can be modulated by maternal diet ${ }^{3,6,7}$. Importantly, however, the extent to which these effects can be inherited by subsequent generations remains to be clarified.

Perhaps even more compelling evidence for repeat sequences contributing to transgenerational epigenetic inheritance has come from data showing that IAPs may be more refractory than other sequences to germline and pre-implantation DNA demethylation ${ }^{29-31}$. These elements therefore have the properties required of sequences for which the epigenetic state can persist transgenerationally and can have an impact on the expression of neighbouring genes. Environmental modulation of the efficiency of repeat sequence reprogramming may contribute to variations in the expression of associated genes in offspring and may lead to phenotypic outcomes.

In addition to the potential contribution of IAPs, other mechanisms for transgenerational epigenetic inheritance may exist, and small RNA-mediated processes, such as those described above in other organisms ${ }^{13,14}$, may be good candidates for involvement ${ }^{3}$. Germline modulation by small RNAs in mammals has particularly been described in the male acting on epigenetic states and post-transcriptionally ${ }^{32,33}$. Defects in this process might have an impact on the behaviour of genes after fertilization. Post-transcriptional regulation induced by small RNAs experimentally injected into germ cells and newly fertilized eggs has been observed, affecting post-fertilization phenotypes ${ }^{34}$. However, how these effects may be propagated into subsequent generations remains a mystery.

"it may be no coincidence that many cases of transgenerational epigenetic inheritance involve repeat sequences"

In the search for mechanisms underlying transgenerational epigenetic inheritance, some emphasis has been placed on genomic imprinting, which is the normal process that causes 
genes to be expressed according to their parental origin. Imprints in mice are regulated by DNA methylation that is established in the germ line but occurs at different loci in the developing sperm and oocyte. What makes imprinted genes such good candidate mediators of transgenerational epigenetic inheritance? First, they are exquisitely dependent on epigenetic mechanisms for their parent-of-origin-specific expression and dosage ${ }^{24}$. Second, imprinted genes function in pathways that contribute to phenotypes that are currently most associated with transgenerational epigenetic effects: growth, metabolism, behaviour and environmental adaptations ${ }^{35}$. Perhaps most importantly, imprints are by definition resistant to the second wave of reprogramming that occurs in the pre-implantation embryo ${ }^{24,27}$. Hence, all that might be required for a defective imprint to have a transgenerational impact is compromised germline reprogramming of methylation imprints.

Recently, experiments have been conducted to test whether imprinted genes as a class are more or less environmentally modulated compared with other genes in a mouse model of intergenerational phenotypic inheritance ${ }^{23}$. Imprinted genes as a group showed no marked difference from randomly selected genes ${ }^{36}$. While some individual imprinted genes showed tissue-specific changes in expression, it is not clear whether the impact on phenotype was a primary or a secondary effect.

Perhaps the strongest argument against transgenerational epigenetic inheritance surrounds the challenge of ruling out genetic effects underlying the phenotype in offspring. The impact of genetic variation on epigenetic state is recognized but is only beginning to be understood and quantified ${ }^{37}$. While tissue-specific differences in epigenetic states within individuals are known to outnumber inter-individual epigenetic differences within the same tissue ${ }^{38}$, a change in epigenetic state between parent and offspring might be caused by genetic variants. As human epidemiological studies are carried out on populations of mixed genetic backgrounds, and also because epigenotype-phenotype analyses can involve small sample sizes, caution in the interpretation of such studies has been encouraged ${ }^{39}$. The relative contributions of genetic and epigenetic variation to transgenerational epigenetic inheritance in mammals will be better understood through the use of isogenic strains in animal studies and the quantitative integration of whole-genome sequence information into epigenotypephenotype analyses on large sample sizes.

Marcus Pembrey. Molecular evidence for transgenerational epigenetic inheritance in humans is limited, but I suspect that it is commonplace. Transgenerational epigenetic inheritance is the best candidate mechanism to explain the male-line transgenerational effects that are now being demonstrated within cohort studies capable of dealing with many social and other confounders ${ }^{40-44}$. If these observations are statistically sound and cannot be explained by genetic or cultural inheritance, then this is transgenerational epigenetic inheritance in its broadest sense.

Humans have imprinted genes (see the Catalogue of Parent of Origin Effects website), and genomic imprinting establishes the principle of transgenerational epigenetic inheritance: the gene is active or silent depending on epigenetic marks placed in the parental generation that survive erasure as they pass to the offspring ${ }^{45,46}$. This parent-of-origin-dependent gene expression reflects a robust, evolved response to differences in cellular conditions between 
egg and sperm formation. Note that this response depends on the DNA sequence of the relevant imprinting control centre, microdeletions of which cause imprinting to fail ${ }^{47}$. More generally, I expect transgenerational epigenetic inheritance to be dependent on the DNA context, but this does not exclude an enduring change in gene expression (without DNA sequence change) in response to paternal or ancestral environmental exposures.

Remarkable data collected from three generations were observed in the Överkalix cohorts ${ }^{40-42,44}$. On the basis of harvest records, variation in the food supply during the early life of paternal grandparents was related to variation in mortality rate (and diabetic deaths; see below) in their grandchildren. There were striking sex-specific transmissions, such that the food supply of the father's father was associated with the mortality rate of just grandsons, while the early-life food supply of the father's mother was associated with the mortality rate of just granddaughters. These different associations were generated through the same set of fathers, making 'cultural inheritance' down to the grandchild generation implausible. The associations also persist on adjusting for the grandchildren's early-life circumstances ${ }^{44}$. If you plot paternal grandfathers against granddaughters or paternal grandmothers against grandsons you get the null hypothesis: namely, that there is absolutely no association ${ }^{20}$. The Överkalix studies also show that there are exposure-sensitive periods (mid-childhood) and exposure-insensitive periods (puberty), making straight genetic inheritance implausible. For the grandmothers, there was an additional sensitive period (namely, from conception to 3 years old) in line with the timing of female gametogenesis.

While these patterns were observed in two of three independent Överkalix cohorts ${ }^{20}$, this population might be exceptional in its responsiveness to these fluctuating circumstances of the nineteenth century. Replications of paternal line transmission using contemporary cohorts have studied only father to off-spring transmission to date. In the UK Avon Longitudinal Study of Parents and Children (ALSPAC) ${ }^{48}$, when using paternal onset of regular smoking as the exposure, we hypothesized that any growth effect on offspring would be confined to paternal smoking before puberty, in line with the Överkalix exposuresensitive period. This is what we found: sons of fathers who started to smoke before 11 years old, but not later, had a greater body mass index (BMI) at 9 years old ${ }^{20}$ (see below for recent unpublished results). Working independently on betel quid Barbara Boucher, working with Tony Chen, replicated what she had previously demonstrated in mice ${ }^{49}$ in the Keelung Community of Taiwan: a dosage-dependent association of paternal betel quid use with early metabolic syndrome in the adult offspring who had never chewed betel quid themselves ${ }^{43}$. These three population studies suggest that male-line transgenerational effects might be widespread.

Susan Lindquist. Since you have asked me about yeast, let me stick to the epigenetic inheritance I know best: prions. First, a definition. Prions are protein-based elements of inheritance 50,51 . They represent 'epigenetics in the extreme' because they are not based on DNA or histone modifications. Prion inheritance operates through the formation and transmission of protein templates. These are usually inherited independently of chromosomes $^{52}$. 
"I predict that this remarkable mechanism for self-perpetuating distinct new biological states will prove to be very common indeed"

Prion proteins have the special property of being able to exist stably in at least two conformational states that have profoundly different structures and functions ${ }^{50,51}$. Of course, many proteins adopt distinct conformations. What is special about prions is their ability to self-template at least one of those conformations to other proteins of the same type in a manner that can serve as a mechanism for inheriting a new biological trait. Once protein $\mathrm{X}$ switches to the prion form, it serves as a template to convert more and more molecules of protein $\mathrm{X}$ into the same prion form. These prion switches are rare compared with rates of cell division, but when they occur, they are very stable ${ }^{51}$. In fact, the prions that we know the most about convert soluble proteins into self-templating amyloids. Amyloids are the most stable protein folds we know.

To ensure that the changes in function brought about by these conformational changes are heritable, the protein templates must be divided and distributed to daughter cells in an orderly and highly predictable way. Prions use specific components of the cellular proteinfolding machinery (remodelling factors such as heat-shock protein 104 (Hsp104) and chaperones such as Hsp70) to do so ${ }^{53}$. In effect, this machinery constitutes a 'mitotic apparatus' for the transmission of the prions, albeit it is a mitotic apparatus that is very different from the microtubules and centromeres employed by DNA.

This protein-based form of inheritance was once considered a freak-show oddity. But recent data indicate that it is actually very common. In Saccharomyces cerevisiae, several proteins that produce heritable traits in this way have been well characterized both genetically and biochemically ${ }^{51}$. At least another dozen have domains that are fully capable of doing so ${ }^{54}$ (although it is not yet certain that they all operate as prions in their normal contexts).

S. cerevisiae is the most genetically tractable organism we know. Prions were first discovered there only fairly recently ${ }^{55,56}$. Not surprisingly, then, this is the only organism in which the phenomenon has been broadly characterized. But there are now many reasons to believe that prions are very commonly used by fungi and, indeed, that all eukaryotes have and take advantage of - prion proteins. First, the genes encoding homologues of some well-characterized prion proteins in very distantly related fungi have been transgenically expressed in S. cerevisiae, where they have the same capacity to serve as protein-based elements of inheritance ${ }^{57,58}$. Second, in surveying $~ 700$ wild yeast strains for protein-based inheritance ${ }^{59}$, we found dozens that carried prions that had previously been characterized only in laboratory strains. And at least one-third of wild strains carried protein-based elements of inheritance with all of the hallmarks of prions. Third, surveys of other eukaryotic genomes have identified many proteins that have the unusual amino acid sequence characteristics of the well-known prions ${ }^{50,51}$. Some contain an enormous number of them. Furthermore, we know that there are prion proteins we have not yet learned to identify by simple bioinformatics ${ }^{60}$. So the number of prion proteins will doubtlessly increase.

As we learn more and more about biological systems, boundaries blur, and the selftemplating, self-assembling properties of the proteins we know as prions are being found in 
many other contexts ${ }^{61-64}$. So I predict that this remarkable mechanism for self-perpetuating distinct new biological states will prove to be very common indeed. In fact, prions are characterized by simple, low-complexity sequences. I would go so far as to bet that they played a major part in the early evolution of life on Earth.

Q How much impact does transgenerational epigenetic inheritance have on organisms?

U.G. One can easily imagine that it would be beneficial for plants, as sessile organisms, to take advantage of the information that they acquire about their environment and to pass it onto their progeny. The likelihood that the progeny will experience a similar environment is rather high, given that seeds are often dispersed locally. Thus, passing on such information to the progeny would have an adaptive value, and epigenetic inheritance is the prime candidate for its transmission. Indeed, plants have an extensive repertoire of epigenetic regulation involving mechanisms based on DNA methylation, histone modification and RNA. This includes a unique epigenetic pathway, RNA-directed DNA methylation, whereby DNA methylation and chromatin modifications are guided in a sequence-specific manner by small RNAs ${ }^{65}$. Such small RNAs can be regulated developmentally or induced by biotic or abiotic stresses ${ }^{66}$, providing great versatility to epigenetic gene regulation and the potential for transmitting acquired information to the progeny. It is not surprising then that the transgenerational epigenetic inheritance of environmentally induced changes has attracted much attention.

Many recent studies have focused on the epigenetic inheritance of responses to biotic and abiotic stresses. Experiments in several species indicate that effects of stress or other environmental conditions might be inherited (for example, see REFS 67-69). For instance, it was reported that stress could induce a hyper-recombination phenotype in transgenic mouseear cress (Arabidopsis thaliana) that persisted for up to four generations without any subsequent stress exposure ${ }^{67}$. Later studies, however, could detect these effects only in the direct progeny of the stressed plants (for example, see REF. 70) or found the variation between experiments to be in the same order of magnitude as the effects induced by stress ${ }^{71}$. In another example, it was shown that stress treatments in dandelions (Taraxacum officinale) can induce methylation changes that are inherited by their progeny ${ }^{69}$. Additional experiments showed, however, that there is a high variability between experiments and that these effects are not always observed ${ }^{72}$.

In summary, there are not yet any clear-cut studies that would unambiguously demonstrate the transgenerational epigenetic inheritance of environmentally induced effects ${ }^{9,10}$. Many studies failed to exclude parental effects, were not sufficiently replicated to distinguish stochastic effects from treatment effects or did not integrate phenotypic characterizations with more detailed molecular and genetic analyses (for a detailed discussion, see REF 9). Thus, although the meiotic inheritance of epialleles has clearly been demonstrated in plants, it is not yet clear whether environmentally induced epigenetic states show transgenerational inheritance. I expect that future studies that integrate multigenerational phenotyping with genome-wide transcriptomic and epigenomic analyses will provide a definite answer to this question. 
W.G.K. The genomic battles between invasive genetic elements and host epigenetic responses have left lasting marks on genome structure and function, but it is interesting to consider whether the piRNA genome defence mechanisms and their impact on transgenerational transposon repression may also be potential players in heritable phenotypic alterations in nature. As mentioned, the piRNA 'catalogue' of potential targets can include genes that are normally expressed, but a self-recognition system is hypothesized to prevent their targeting. An imbalance in the self versus non-self antagonism in the initial recognition pathways could theoretically engage the targeting and heritable repression of any locus. Such imbalances could be created by temporary environmental insults or even transient exposure to an altered genotype; that is, both nature and nurture are capable of producing transgenerationally stable phenotypic outcomes.

"the transgenerational stability of any novel epigenetic state at least partly depends on its resistance to reprogramming"

There is evidence that transient exposure to altered genotype can produce transgenerational phenotypes in C. elegans. Defects in a form of histone methylation maintenance machinery, which involves the conserved MLL complex, lead to heritable increases in longevity in $C$. elegans $^{73}$. Surprisingly, loss of the components in the germ line of one generation causes increased longevity for multiple generations, even after restoration of MLL complex activity. The effect, however, is strikingly limited to several generations, after which normal lifespan reappears. Given that metabolic rate is inversely correlated with lifespan, one possibility is that the loss of some histone methylation maintenance weakens the 'memory' of metabolic gene activity in the germ cells, and this results in decreased transcription of these loci in all tissues in subsequent generations. The re-establishment of robust transcription of metabolic genes, and hence reappearance of normal lifespan, may require several generations of reiterative transcription coupled with restored memory maintenance. Notably, a persistent lack of MLL complex activity in several generations results in a depletion of germline stem cells and eventually highly penetrant sterility: a 'mortal germ line' phenotype ${ }^{74,75}$.

An argument against any potential lasting impact of epigenetic phenomena is that many of the processes are dynamic, and even the self-reinforcing or maintenance mechanisms have some inherent variability, hence the observed metastability of transgenerational phenomena. This stochastic variation may arise in many ways, but chief among them is probably threshold effects. In considering the intertwined RNA interference (RNAi)- and chromatinbased mechanisms described above, the robustness of each overlapping mechanism can potentially affect the robustness of other mechanisms, and some sum of the robustness of all steps could generate outcomes with different degrees of transgenerational stability. For example, the generation of secondary small RNAs in the piRNA system could have quantitative variability, which may affect the efficiency of the establishment of stable chromatin states, and these states may themselves be subject to variable maintenance and heritability. Furthermore, the epigenome established in the parent and inherited between generations via the gametes encounters 'reprogramming' mechanisms in the zygote. Thus, the transgenerational stability of any novel epigenetic state at least partly depends on its resistance to reprogramming. The degree of reprogramming could depend on the efficiency 
of the reprogramming mechanism, which may be influenced by the density of epigenetic information at a locus. The loss of a histone $\mathrm{H} 3$ demethylase in C. elegans, for example, eventually causes a mortal germ line phenotype, correlating with a rise in its target modification, but the penetrance of the sterility, the increased accumulation of histone methylation and the transcriptional defects play out over dozens of generations before reaching a 'crisis generation'. Importantly, even at the crisis generation, some animals escape sterility, yet these animals and their descendants still recapitulate the mortal germline dynamics over subsequent generations ${ }^{76}$. Thus, any novel epigenetic information has numerous obstacles to traverse to become established; chief among these obstacles is the constraint of compatibility with fertility.

However, stable transgenerational phenomena are observed, and such processes might be useful. It is possible that the plasticity of epigenetic states could open doors of opportunity in changing environments. If one considers the metastability and stochasticity of transgenerational epigenetic inheritance, it is possible to envision a Lamarckian-type edge that epialleles could provide during environmental stress. For example, an epigenetic change in expression of a gene that is favoured in a new environment allows a metastable adaptation to the new environment. The metastability may provide a hedge bet that the old environmental conditions will return, yet in the meantime it may produce enough subsequent generations to sample more stable genetic alterations for adaptive usefulness.

A.C.F.-S. If an epigenetic mechanism regulating transgeneration inheritance exists in mammals, then this has profound implications for how we consider inheritance, for mechanisms underlying diseases and for phenotypes that are regulated by gene-environment interactions.

The windows of opportunity for such mechanisms to take effect in mammals lie in the germ line and during pre-implantation development. But how many generations might be affected? Underlying this question is the concept of intergenerational (that is, shorter-term transgenerational effects) versus transgenerational effects over multiple generations ${ }^{27}$. For example, consider the impact of a compromised in utero environment on the developing fetus. This fetus, once born, might exhibit phenotypes as a consequence of its in utero exposure. Furthermore, because its developing germ cells were also exposed, the offspring of that individual might also exhibit defective phenotypes. However, unless that individual (and its germ cells) is exposed to further insult, it would be unlikely for a further generation to be affected. Hence, phenotypic inheritance might cease, and this might be considered to be a shorter-term intergenerational effect rather than a long-term transgenerational effect. By contrast, if the phenotype were indeed transmitted to a third generation, then this would be true transgenerational inheritance: a process that was previously attributed to genetic effects, that is harder to attribute to an epigenetic mechanism and that is the subject of considerable debate in the field because it rewrites the rules of heritability. One aspect perhaps worth considering is the fact that DNA methylation is a mutagen that contributes to $\mathrm{C}$ to $\mathrm{T}$ transitions if not repaired. Indeed, epigenetic modification mediating such mutation may underlie long-term genetic inactivation of transposable elements ${ }^{77}$. Hence, a short-term epigenetic change, leading to a genetic mutation with long-term effects, may have the potential to contribute to a form of transgenerational epigenetic inheritance. 
M.P. As indicated above, transgenerational epigenetic inheritance is the best candidate mechanism for the male-line transgenerational effects that have been documented so far in humans. The Överkalix data ${ }^{20}$ suggest that an existing, perhaps adaptive, mechanism is being induced, so I favour the term transgenerational response (TGR) rather than transgenerational effect. In terms of the impact on descendants, there are two important points to make. First, the impact may be a protective adaptation that maintains health in the face of continuing adverse exposure down the generations. Such adaptation has been demonstrated in the remarkable experiments that have been done in male rats on ancestral liver damage, leading to heritable, epigenetically mediated reprogramming of hepatic wound healing ${ }^{78}$.

"The effect sizes seen for human TGRs to date are considerable by genetic epidemiology standards"

How important transgenerational epigenetic inheritance is in humans may have to be measured in terms of resilience as well as disease. Second, the focus is not inheritance of acquired characteristics or 'phenotypic transmission', for which population evidence has been sought with little success, except perhaps in the case of maternal transmission of diabetes. Lamarck conflated the evolutionary process with adaptation and, in my view, human TGRs are likely to be primarily a reflection of adaptive responses in action, although the response systems themselves will have evolved by DNA sequence change and natural selection $^{46,79}$.

The effect sizes seen for human TGRs to date are considerable by genetic epidemiology standards, with general mortality rate ratios ranging from 0.4 to 2.8 in the grandchildren, depending on ancestral food supply ${ }^{20}$. The association of the paternal grandfather's good food supply in mid-childhood with diabetic deaths in the grandchildren gave an odds ratio of 4.1 (95\% confidence interval: 1.33, 12.93) - quite a shock! Admittedly, it was based on just 19 affected grandchildren (each with a different grandfather), but this was testing a prior hypothesis ${ }^{41,42}$, as indeed most steps in the human TGR trail have been over the last decade ${ }^{80}$. Clearly, replication is needed before drawing firm conclusions, but replication of historical studies is difficult. Contemporary cohort studies, such as ALSPAC, that can adjust for many confounders may offer better opportunities to test TGR in a rigorous way. Unpublished follow-up results from ALSPAC show that paternal onset of regular smoking before 11 years of age is associated with increased mean BMI and body fat mass in sons throughout their development from 11-17 years of age, even though these fathers themselves do not have increased adiposity. These sons are carrying an extra 5-10 kg of fat mass compared with the sons of all other fathers (K. Northstone, J. Golding, L. Miller and M.P., unpublished observations). While the Taiwan study is not directly comparable (paternal betel exposure is from 18 years; B. Boucher, Institute of Cell and Molecular Science, Queen Mary, University of London, personal communication) exposure increases the risk of early manifestations of the metabolic syndrome in offspring 2.53 -fold (95\% confidence interval: $1.03,2.64)$ compared with paternal non-exposure ${ }^{43}$.

Smoking and betel quid chewing are major public health concerns worldwide, and as they are common, it should allow independent replication of the transgenerational effects. In 2002, I wrote a commentary entitled 'Time to take epigenetic inheritance seriously' ${ }^{81}$. Ten 
years on, I regard understanding human TGR as crucial, even if the eventual mechanisms do not meet the definition of transgenerational epigenetic inheritance that the purists demand!

S.L. The impact is enormous, in part because prions change cell biology in many, many different ways. First, the prions discovered to date are mostly proteins that regulate 'information flow' in the cell: transcription factors, translation factors, RNA-binding proteins and signal transducers ${ }^{51,54}$. This means that when a single protein switches into the prion state, it creates a new trait that is both immediately heritable and biologically complex in nature.

One of the prion proteins we have worked on is a transcriptional repressor known as $[\mathrm{MOT} 3+]^{54}$. When it switches into to the prion state, it is sequestered from the chromosome. Many genes are simultaneously de-repressed. Because Mot3 regulates genes that are involved in anaerobic growth, adhesion and filamentation, among others, the biological properties of cells carrying this prion are very different from those of genetically identical cells that do not carry the prion ${ }^{82}$. This is one of the prions that we have found being used in wild yeast strains ${ }^{59}$. Another prion that we have been studying changes a fundamental aspect of carbon source utilization ${ }^{83}$. Humans use $S$. cerevisiae to produce alcohol because - as long as glucose is around - it ferments the sugar to alcohol. The cells will not use any of the alcohol, or any other carbon source, until glucose is exhausted. This trait (glucose repression) can be controlled by prion proteins. When they switch to the prion state, cells can suddenly get around glucose repression ${ }^{83}$. This is a pretty fundamental metabolic switch. We recently found that this prion too appears commonly in wild strains, allowing cells to respond to their ecological niche in a heritable way (D. F. Jarosz, J. C. S. Brown, G. A. Walker, A. K. Lancaster, L. F. Bisson and S.L., unpublished observations). Other prions alter the activity of chromatin-remodelling factors ${ }^{54,84}$, prevent fungi from out-crossing ${ }^{85}$ or alter antibiotic resistance ${ }^{60,86}$. In other words, they alter the biology of the organism in fundamental ways.

One of the most interesting aspects of prion biology is their ability to serve as 'bet hedging' factors $^{52}$. Microorganisms are responsible for most of the biodiversity on Earth. They survive in fluctuating environments very well, and prions contribute to this, we believe. Because prions are embodied by a change in protein folding, stresses that alter protein homeostasis can induce prion switching. This provides a robust mechanism for the inheritance of environmentally acquired traits.

Let us take, as one example, a prion formed by the essential translation termination factor Sup35. In its prion form, Sup35 is sequestered from the ribosome, and ribosomes then begin to read through stop codons. In certain cases, so much Sup35 is sequestered that cells die. Some argue, therefore, that this prion is simply a 'protein folding disease' of yeast cells ${ }^{87}$. However, the Sup35 protein more commonly establishes an equilibrium between the prion and nonprion states that results in only a moderate readthrough ${ }^{50,51}$. The prion-induced change in gene expression then creates a host of new heritable traits, and a substantial number of these are beneficial ${ }^{86}$. Because this prion uncovers previously hidden genetic information on multiple mRNAs at once, it immediately creates heritable traits that would take a great deal longer to acquire by successive individual mutations. We think that this 
prion, and others, cannot only promote survival in fluctuating environments but can serve as an engine for the evolution of new traits ${ }^{52,86}$.

\section{Acknowledgments}

U.G. is supported by the Swiss National Science Foundation and the European Research Council. W.G.K. acknowledges J. Lucchesi and S. Battacharyya for helpful discussions and comments. A.C.F.-S. is currently funded by the UK Medical Research Council (MRC), the Wellcome Trust and the EUFP7 programmes EpigeneSys, BLUEPRINT, EpiHealth and INGENIUM. S.L. thanks G. Newby for helpful comments.

\section{References}

1. Cloud, J. Why your DNA isn't your destiny. Time. 2010. [online], http://www.time.com/time/ magazine/article/0,9171,1952313,00.html

2. Hauser MT, et al. Transgenerational epigenetic inheritance in plants. Biochim. Biophys. Acta. 2011; 1809:459-468. [PubMed: 21515434]

3. Daxinger L, Whitelaw E. Understanding transgenerational epigenetic inheritance via the gametes in mammals. Nature Rev. Genet. 2012; 13:153-162. [PubMed: 22290458]

4. Riggs, VEA.; Martienssen, RA.; Riggs, AD., editors. Epigenetic Mechanisms of Gene Regulation. Cold Spring Harbor Laboratory Press; 1996.

5. Morgan HD, et al. Epigenetic inheritance at the agouti locus in the mouse. Nature Genet. 1999; 23:314-318. [PubMed: 10545949]

6. Cropley JE, et al. Germ-line epigenetic modification of the murine $A^{v y}$ allele by nutritional supplementation. Proc. Natl Acad. Sci. USA. 2006; 103:17308-17312. [PubMed: 17101998]

7. Waterland RA, et al. Diet-induced hypermethylation at agouti viable yellow is not inherited transgenerationally through the female. FASEB J. 2007; 21:3380-3385. [PubMed: 17551099]

8. Grossniklaus U. Plant germline development: a tale of cross-talk, signaling, and cellular interactions. Sex. Plant Reprod. 2011; 24:91-95. [PubMed: 21590362]

9. Pecinka A, Mittelsten Scheid O. Stress-induced chromatin changes: a critical view on their heritability. Plant Cell Physiol. 2012; 53:801-808. [PubMed: 22457398]

10. Paszkowski J, Grossniklaus U. Selected aspects of transgenerational epigenetic inheritance and resetting in plants. Curr. Opin. Plant Biol. 2011; 14:195-203. [PubMed: 21333585]

11. Cubas $P$, et al. An epigenetic mutation responsible for natural variation in floral symmetry. Nature. 1999; 401:157-161. [PubMed: 10490023]

12. Manning K, et al. A naturally occurring epigenetic mutation in a gene encoding an SBP-box transcription factor inhibits tomato fruit ripening. Nature Genet. 2006; 38:948-952. [PubMed: 16832354]

13. Shirayama M, et al. piRNAs initiate an epigenetic memory of nonself RNA in the C. elegans germline. Cell. 2012; 150:65-77. [PubMed: 22738726]

14. Ashe A, et al. piRNAs can trigger a multigenerational epigenetic memory in the germline of $C$. elegans. Cell. 2012; 150:88-99. [PubMed: 22738725]

15. Rechtsteiner A, et al. The histone H3K36 methyltransferase MES-4 acts epigenetically to transmit the memory of germline gene expression to progeny. PLoS Genet. 2010; 6:e1001091. [PubMed: 20824077]

16. Furuhashi H, et al. Trans-generational epigenetic regulation of C. elegans primordial germ cells. Epigenet. Chromatin. 2010; 3:15.

17. Gaydos LJ, et al. Antagonism between MES-4 and Polycomb repressive complex 2 promotes appropriate gene expression in C. elegans germ cells. Cell Rep. 2012; 2:1169-1177. [PubMed: 23103171]

18. Gassmann R, et al. An inverse relationship to germline transcription defines centromeric chromatin in C. elegans. Nature. 2012; 484:534-537. [PubMed: 22495302]

19. Nadeau JH. Transgenerational genetic effects on phenotypic variation and disease risk. Hum. Mol. Genet. 2009; 18:R202-R210. [PubMed: 19808797] 
20. Pembrey ME, et al. Sex-specific, male-line transgenerational responses in humans. Eur. J. Hum. Genet. 2006; 14:159-166. [PubMed: 16391557]

21. Ng SF, et al. Chronic high-fat diet in fathers programs $\beta$-cell dysfunction in female rat offspring. Nature. 2010; 467:963-966. [PubMed: 20962845]

22. Carone BR, et al. Paternally induced transgenerational environmental reprogramming of metabolic gene expression in mammals. Cell. 2010; 143:1084-1096. [PubMed: 21183072]

23. Jiminez-Chirallon JC, et al. Intergenerational 'programming' of glucose intolerance and obesity by in utero undernutrition. Diabetes. 2009; 58:460-468. [PubMed: 19017762]

24. Ferguson-Smith AC. Genomic imprinting: the emergence of an epigenetic paradigm. Nature Rev. Genet. 2011; 12:565-575. [PubMed: 21765458]

25. Ohhata T, Wutz A. Reactivation of the inactive X chromosome in development and reprogramming. Cell Mol Life Sci. Sep 30.2012 doi:10.1007/s00018-012-1174-3.

26. Sasaki H, Matsui Y. Epigenetic events in mammalian germ-cell development: reprogramming and beyond. Nature Rev. Genet. 2008; 9:129-140. [PubMed: 18197165]

27. Ferguson-Smith AC, Patti ME. You are what your dad ate. Cell. Metab. 2011; 13:115-117. [PubMed: 21284975]

28. Fazzari MJ, Greally JM. Epigenomics: beyond CpG islands. Nature Rev. Genet. 2004; 5:446-455. [PubMed: 15153997]

29. Hackett JA, et al. Germline DNA demethylation dynamics and imprint erasure through 5hydroxymethylcytosine. Science. 2012; 339:448-452. [PubMed: 23223451]

30. Lane N, et al. Resistance of IAPs to methylation reprogramming may provide a mechanism for epigenetic inheritance in the mouse. Genesis. 2003; 35:88-93. [PubMed: 12533790]

31. Seisenberger $\mathrm{S}$, et al. The dynamics of genome-wide DNA methylation reprogramming in mouse primordial germ cells. Mol. Cell. 2012; 48:849-862. [PubMed: 23219530]

32. Watanabe T, et al. Role for piRNAs and noncoding RNA in de novo DNA methylation of the imprinted mouse Rasgrf1 locus. Science. 2011; 332:848-852. [PubMed: 21566194]

33. Vourekas A, et al. Mili and Miwi target RNA repertoire reveals piRNA biogenesis and function of Miwi in spermiogenesis. Nature Struct. Mol. Biol. 2012; 19:773-781. [PubMed: 22842725]

34. Wagner KD, et al. RNA induction and inheritance of epigenetic cardiac hypertrophy in the mouse. Dev. Cell. 2008; 14:962-969. [PubMed: 18539123]

35. Charalambous M, da Rocha ST, Ferguson-Smith AC. Genomic imprinting, growth control and the allocation of nutritional resources: consequences for postnatal life. Curr. Opin. Endocrinol. Diabetes Obes. 2007; 14:3-12. [PubMed: 17940412]

36. Radford EJ, et al. An unbiased assessment of the role of imprinted genes in an intergeneration model of developmental programming. PLoS Genet. 2012; 8:e1002605. [PubMed: 22511876]

37. Ward LD, Kellis M. Interpreting noncoding genetic variation in complex traits and human disease. Nature Biotech. 2012; 30:1095-1106.

38. Davies MN, et al. Functional annotation of the human brain methylome identifies tissue-specific epigenetic variation across brain and blood. Genome Biol. 2012; 13:R43. [PubMed: 22703893]

39. Heijmans BT, Mill J. Commentary: the seven plagues of epigenetic epidemiology. Int. J. Epidemiol. 2012; 41:74-78. [PubMed: 22269254]

40. Bygren LO, Kaati G, Edvinsson S. Longevity determined by ancestors' over nutrition during their slow growth period. Acta Biotheoret. 2001; 49:53-59.

41. Kaati G, Bygren LO, Edvinsson S. Cardiovascular and diabetes mortality determined by nutrition during parents' and grandparents' slow growth period. Eur. J. Hum. Genet. 2002; 10:682-688. [PubMed: 12404098]

42. Bygren LO, Kaati G, Edvinsson S, Pembrey ME. Reply to Senn. Eur. J. Hum. Genet. 2006; 14:1149-1150. [PubMed: 16823395]

43. Chen TH, Chiu YH, Boucher BJ. Transgenerational effects of betel-quid chewing on the development of the metabolic syndrome in the Keelung Community-based Integrated Screening Program. Am J. Clin. Nutr. 2006; 83:688-692. [PubMed: 16522918]

44. Kaati G, Bygren LO, Pembrey M, Sjostrom M. Transgenerational response to nutrition, early life circumstances and longevity. Eur. J. Hum. Genet. 2007; 15:784-790. [PubMed: 17457370] 
45. Reik W, Walter J. Genomic imprinting: parental influence on the genome. Nature Rev. Genet. 2001; 2:21-32. [PubMed: 11253064]

46. Pembrey ME. Imprinting and transgenerational modulation of gene expression; human growth as a model. Acta Genet. Med. Gemellol. 1996; 45:111-125. [PubMed: 8872020]

47. Buiting K, et al. Disruption of the bipartite imprinting centre in a family with Angelman syndrome. Am. J. Hum. Genet. 2001; 68:1290-1294. [PubMed: 11283796]

48. Golding J, Pembrey M, Jones R, The ALSPAC Study Team. ALSPAC-The Avon Longitudinal Study of Parents and Children. Paediatr. Perinat. Epidemiol. 2001; 15:74-87. [PubMed: 11237119]

49. Boucher BJ, Ewen SW, Stowers JM. Betel nut (Areca catechu) consumption and the induction of glucose intolerance in adult CD1 mice and in their F1 and F2 offspring. Diabetologia. 1994; 37:49-55. [PubMed: 8150230]

50. Shorter J, Lindquist S. Prions as adaptive conduits of memory and inheritance. Nature Rev. Genet. 2005; 6:435-450. [PubMed: 15931169]

51. Liebman SW, Chernoff YO. Prions in yeast. Genetics. 2012; 191:1041-1072. [PubMed: 22879407]

52. Halfmann R, Lindquist S. Epigenetics in the extreme: prions and the inheritance of environmentally acquired traits. Science. 2010; 330:629-632. [PubMed: 21030648]

53. Shorter J, Lindquist S. Hsp104, Hsp70 and Hsp40 interplay regulates formation, growth and elimination of Sup35 prions. EMBO J. 2008; 27:2712-2724. [PubMed: 18833196]

54. Alberti S, Halfmann R, King O, Kapila A, Lindquist S. A systematic survey identifies prions and illuminates sequence features of prionogenic proteins. Cell. 2009; 137:146-158. [PubMed: 19345193]

55. Wickner RB. [URE3] as an altered URE2 protein: evidence for a prion analog in Saccharomyces cerevisiae. Science. 1994; 264:622-626.

56. Patino MM, Liu J, Glover JR, Lindquist S. Support for the prion hypothesis for inheritance of a phenotypic trait in yeast. Science. 1996; 273:622-626. [PubMed: 8662547]

57. Santoso A, Chien P, Osherovich LZ, Weissman JS. Molecular basis of a yeast prion species barrier. Cell. 2000; 100:277-288. [PubMed: 10660050]

58. Nakayashiki T, Ebihara K, Bannai H, Nakamura Y. Yeast [PSI+] "prions” that are crosstransmissible and susceptible beyond a species barrier through a quasi-prion state. Mol. Cell. 2001; 7:1121-1130. [PubMed: 11430816]

59. Halfmann R, et al. Prions are a common mechanism for phenotypic inheritance in wild yeasts. Nature. 2012; 482:363-368. [PubMed: 22337056]

60. Suzuki G, Shimazu N, Tanaka M. A yeast prion, Mod5, promotes acquired drug resistance and cell survival under environmental stress. Science. 2012; 336:355-359. [PubMed: 22517861]

61. Si K, Lindquist S, Kandel ER. A neuronal isoform of the aplysia CPEB has prion-like properties. Cell. 2003; 115:879-891. [PubMed: 14697205]

62. Kato M, et al. Cell-free formation of RNA granules: low complexity sequence domains form dynamic fibers within hydrogels. Cell. 2012; 149:753-767. [PubMed: 22579281]

63. Brangwynne CP, et al. Germline P granules are liquid droplets that localize by controlled dissolution/condensation. Science. 2009; 324:1729-1732. [PubMed: 19460965]

64. Hou F, et al. MAVS forms functional prion-like aggregates to activate and propagate antiviral innate immune response. Cell. 2011; 146:448-461. [PubMed: 21782231]

65. Law JA, Jacobsen SE. Establishing, maintaining and modifying DNA methylation patterns in plants and animals. Nature Rev. Genet. 2012; 11:204-220. [PubMed: 20142834]

66. Khraiwesh B, et al. Role of miRNAs and siRNAs in biotic and abiotic stress responses of plants. Biochim. Biophys. Acta. 2012; 1819:137-148. [PubMed: 21605713]

67. Molinier J, et al. Trasngeneration memory of stress in plants. Nature. 2006; 442:1046-1049. [PubMed: 16892047]

68. Whittle CA, et al. Adaptive epigenetic memory of ancestral temperature regime in Arabidopsis thaliana. Botany. 2009; 87:650-657. 
69. Verhoeven KJF, et al. Stress-induced DNA methylation changes and their heritability in asexual dandelions. New Phytol. 2010; 185:1108-1118. [PubMed: 20003072]

70. Boyko A, et al. Transgenerational adaptation of Arabidopsis to stress requires DNA methylation and the function of DICER-LIKE proteins. PLoS ONE. 2010; 5:e9514. [PubMed: 20209086]

71. Pecinka A, et al. Transgenerational stress memory is not a general response in Arabidopsis. PLoS ONE. 2009; 4:e5202. [PubMed: 19381297]

72. Verhoeven KJF, et al. Transgenerational effects of stress exposure on offspring phenotypes in apomictic dandelion. PLoS ONE. 2012; 6:e38605. [PubMed: 22723869]

73. Greer EL, et al. Transgenerational epigenetic inheritance of longevity in Caenorhabditis elegans. Nature. 2011; 479:365-371. [PubMed: 22012258]

74. Simonet T, et al. Antagonistic functions of SET-2/SET1 and HPL/HP1 proteins in C. elegans development. Dev. Biol. 2007; 312:367-383. [PubMed: 17967446]

75. Li T, Kelly WG. A role for Set1/MLL-related components in epigenetic regulation of the Caenorhabditis elegans germ line. PLoS Genet. 2011; 7:e1001349. [PubMed: 21455483]

76. Katz DJ, et al. A C. elegans LSD1 demethylase contributes to germline immortality by reprogramming epigenetic memory. Cell. 2009; 137:308-320. [PubMed: 19379696]

77. Walsh CP, Xu GL. Cytosine methylation and DNA repair. Curr. Top. Microbiol. Immunol. 2006; 301:283-315. [PubMed: 16570853]

78. Zeybel M, et al. Multigenerational epigenetic adaptation of the hepatic wound-healing response. Nature Med. 2012; 18:1369-1377. [PubMed: 22941276]

79. Feinberg AP, Irizarry RA. Stochastic epigenetic variation as a driving force of development, evolutionary adaptation, and disease. Proc. Natl Acad. Sci. USA. 2010; 107(Suppl. 1):1757-1764. [PubMed: 20080672]

80. Pembrey ME. Male-line transgenerational responses in humans. Hum. Fertil. 2010; 13:268-271.

81. Pembrey ME. Time to take epigenetic inheritance seriously. Eur. J. Hum. Genet. 2002; 10:669671. [PubMed: 12404095]

82. Holmes DL, Lancaster AK, Lindquist S, Halfmann R. Heritable remodeling of yeast multicellularity by an environmentally responsive prion. Cell. in the press.

83. Brown JCS, Lindquist S. A heritable switch in carbon source utilization driven by an unual yeast prion. Genes Dev. 2009; 23:2320-2332. [PubMed: 19797769]

84. Crow ET, Du Z, Li L. A small, glutamine-free domain propagates the $\left[\mathrm{SWI}^{+}\right]$prion in budding yeast. Mol. Cell. Biol. 2011; 31:3436-3444. [PubMed: 21670156]

85. Saupe SJ. The [Het-s] prion of Podospora anserina and its role in heterokaryon incompatibility. Sem. Cell Dev. Biol. 2011; 22:460-468.

86. True HL, Lindquist S. A yeast prion provides a mechanism for genetic variation and phenotypic diversity. Nature. 2000; 407:477-483. [PubMed: 11028992]

87. McGlinchey RP, Kryndushkin D, Wickner RB. Suicidal [PSI+] is a lethal yeast prion. Proc. Natl Acad. Sci, USA. 2011; 108:5337-5341. [PubMed: 21402947] 